\title{
A pooled analysis of transarterial radioembolization with yttrium-90 microspheres for the treatment of unresectable intrahepatic cholangiocarcinoma
}

This article was published in the following Dove Press journal: OncoTargets and Therapy

\section{Yanhua Zhen (ID) \\ Bin Liu (D) \\ Zhihui Chang (D) \\ Haiyan Ren (D) \\ Zhaoyu Liu (D) \\ Jiahe Zheng (D)}

Department of Radiology, Shengjing Hospital of China Medical University, Shenyang I 10004, People's Republic of China
Correspondence: Jiahe Zheng

Department of Radiology, Shengjing Hospital of China Medical University, 36, Sanhao Street, Heping District, Shenyang, People's Republic of China

Tel +86 I894025 6027

Fax +862423929902

Email zhengjh120624@|26.com
Purpose: The aim of this pooled analysis was to evaluate the clinical efficacy and safety of transarterial radioembolization (TARE) with yttrium-90 $\left({ }^{90} \mathrm{Y}\right)$ microspheres for the treatment of unresectable intrahepatic cholangiocarcinoma (ICC).

Methods: We searched the Cochrane Library, Embase, PubMed, SCI with the English language from inception to October 2018. A pooled analysis was conducted using Stata software.

Results: There were 16 eligible studies included in this pooled analysis. The pooled median overall survival (OS) from 12 studies was 14.3 (95\% CI: 11.9-17.1) months. Based on Response Evaluation Criteria in Solid Tumors (RECIST), no complete response was reported, and the median of partial response, stable disease and progressive disease were 11.5\% (range: $4.8-35.3 \%$ ), 61.5\% (range: 42.9-81.3\%) and $22.7 \%$ (range: $12.5-52.4 \%$ ) respectively. The pooled disease control rate (DCR) from nine studies was $77.2 \%(95 \%$ CI: 70.2-84.2\%). According to the type of microspheres, subgroup analysis was performed, the median OS in the glass microspheres group was 14.0 (95\% CI: 9.1-21.4) months, and 14.3 (95\% CI: 11.5-17.8) months in the resin microspheres group. The DCR was $77.3 \%$ (95\% CI: $63.5-91.1 \%$ ) and $77.4 \%$ (95\% CI: 66.8-87.9\%) in the glass and resin microspheres groups respectively. Most of the side effects reported in the included studies were mild and did not require intervention.

Conclusion: TARE with ${ }^{90} \mathrm{Y}$ microspheres is safe and effective for patients with unresectable ICC with acceptable side effects. And it seems that the type of microsphere has no influence on therapeutic efficacy.

Keywords: transarterial radioembolization, yttrium-90 microspheres, intrahepatic cholangiocarcinoma, pooled analysis

\section{Introduction}

Intrahepatic cholangiocarcinoma (ICC) is a highly invasive malignancy of the biliary tract with high mortality due to its infiltrative nature, propensity for advanced disease presentation and resistance to chemotherapy. ${ }^{1,2}$ From diagnosis, the median overall survival (OS) of ICC without treatment is about 4.5 months. Surgical resection may be the only potentially curative treatment, however, only $30-40 \%$ of ICC patients are the surgical candidate when the diagnosis is first confirmed. ${ }^{3,4}$ Systemic chemotherapy with cisplatin plus gemcitabine is also limited 
by poor response rates. ${ }^{5,6}$ Transarterial radioembolization (TARE) with yttrium-90 $\left({ }^{90} \mathrm{Y}\right)$-labeled glass or resin microspheres are being used increasingly in primary and secondary liver malignancies, which provides an advantage to the median OS with good tolerance. ${ }^{7,8}$ Al-Adra et $\mathrm{al}^{9}$ reviewed 12 studies regarding TARE with ${ }^{90} \mathrm{Y}$ microspheres for the treatment of unresectable ICC in 2014; there are emerging studies on TARE with ${ }^{90} \mathrm{Y}$ microspheres for the treatment of ICC, so it is necessary to further systematically evaluate the outcomes of TARE with ${ }^{90} \mathrm{Y}$ microspheres in these patients. The aim of this pooled analysis was to comprehensively evaluate the therapeutic efficacy and safety of TARE with ${ }^{90} \mathrm{Y}$ microspheres for the treatment of unresectable ICC.

\section{Material and methods}

\section{Search strategy}

We searched the Cochrane Library, Embase, PubMed, SCI with English language from inception to October 2018. Relevant documents were supplemented by references of retrieved articles. The terms we used to search were related to intrahepatic cholangiocarcinoma, intrahepatic bile duct carcinoma, cholangiocellular carcinoma, neoplasms of the biliary tract, cholangiohepatoma, yttrium90, Y90, ${ }^{90} \mathrm{Y}$, SIR-Spheres, TheraSphere, radiation lobectomy.

Inclusion and exclusion criteria

1. Clinical trials or studies

2. Studies that described TARE with ${ }^{90} \mathrm{Y}$ microspheres in the treatment of unresectable ICC

Exclusion criteria

1. Review articles, animal studies, abstracts, case reports

2. Duplicated clinical studies

3. Studies with fewer than 10 cases

The quality of the studies was independently evaluated by two reviewers based on the Downs and Black quality assessment checklist. ${ }^{10}$

\section{Data extraction}

Two authors extracted the data and a third one resolved any disagreements. The extracted data included details of type of researches (prospective or retrospective cohort), number of patients, age, sex, Eastern Cooperative Oncology Group (ECOG) score, extrahepatic metastases, pre- and postchemotherapy, type of microspheres, dosimetric calculation, follow-up time, median OS, 1-year survival, evaluation criteria, tumor response, side effects (eg clinical toxicities such as fatigue, abdominal pain, nausea, and biochemical toxicities such as decreased albumin, elevated bilirubin, alkaline phosphatase, etc).

\section{Statistical analysis}

Only median OS and disease control rate (DCR) were pooled analysis by Stata 11.0 (StataCorp. 2009. Stata Statistical Software: Release 11. College Station, TX: StataCorp LP.), while other outcomes were analyzed in descriptive statistics. The $\mathrm{I}^{2}$ measure was used to show the inconsistency between studies. An Egger test was used to assess publication bias, and Metaninf was used for sensitivity analysis, a two-sided $P<0.05$ was regarded as significant.

\section{Results}

According to the inclusion and exclusion criteria, 16 eligible studies ${ }^{11-26}$ were identified that reported the TARE with ${ }^{90} \mathrm{Y}$ microspheres for unresectable ICC (Figure 1). Five prospective and 11 retrospective studies were included. There were 472 patients included in this pooled analysis. Patient characteristics were presented in Table 1. Extrahepatic metastases were observed in a median of $48.7 \%$ (range: $8.7-57.9 \%$ ). A median of $71.9 \%$ (range: 0.0-100.0\%) patients received systemic chemotherapy before TARE with ${ }^{90} \mathrm{Y}$ microspheres, and a median of $12.3 \%$ (range: $7.1-28.0 \%$ ) received postoperative chemotherapy.

Table 2 summarized information about the therapeutic outcomes of TARE with ${ }^{90} \mathrm{Y}$ microspheres for ICC. The pooled median OS from 12 studies was 14.3 (95\%CI: 11.9-17.1) months (Figure 2). The tumor response at 3 months after TARE with ${ }^{90} \mathrm{Y}$ microspheres was evaluated according to Response Evaluation Criteria in Solid Tumors (RECIST), no complete response was reported, and the median of partial response, stable disease, progressive disease was $11.5 \%$ (range: $4.8-35.3 \%$ ), 61.5\% (range: $42.9-81.3 \%$ ), $22.7 \%$ (range: $12.5-52.4 \%$ ) respectively. The pooled DCR from available studies was $77.2 \%$ (95\%CI: 70.2-84.2\%) (Figure 3). Subgroup analysis was conducted by microspheres type, the median OS in the glass microspheres group was 14.0 (95\%CI: 9.1-21.4) months, and 14.3 (95\% CI: 11.5-17.8) months in the resin microspheres group. The 


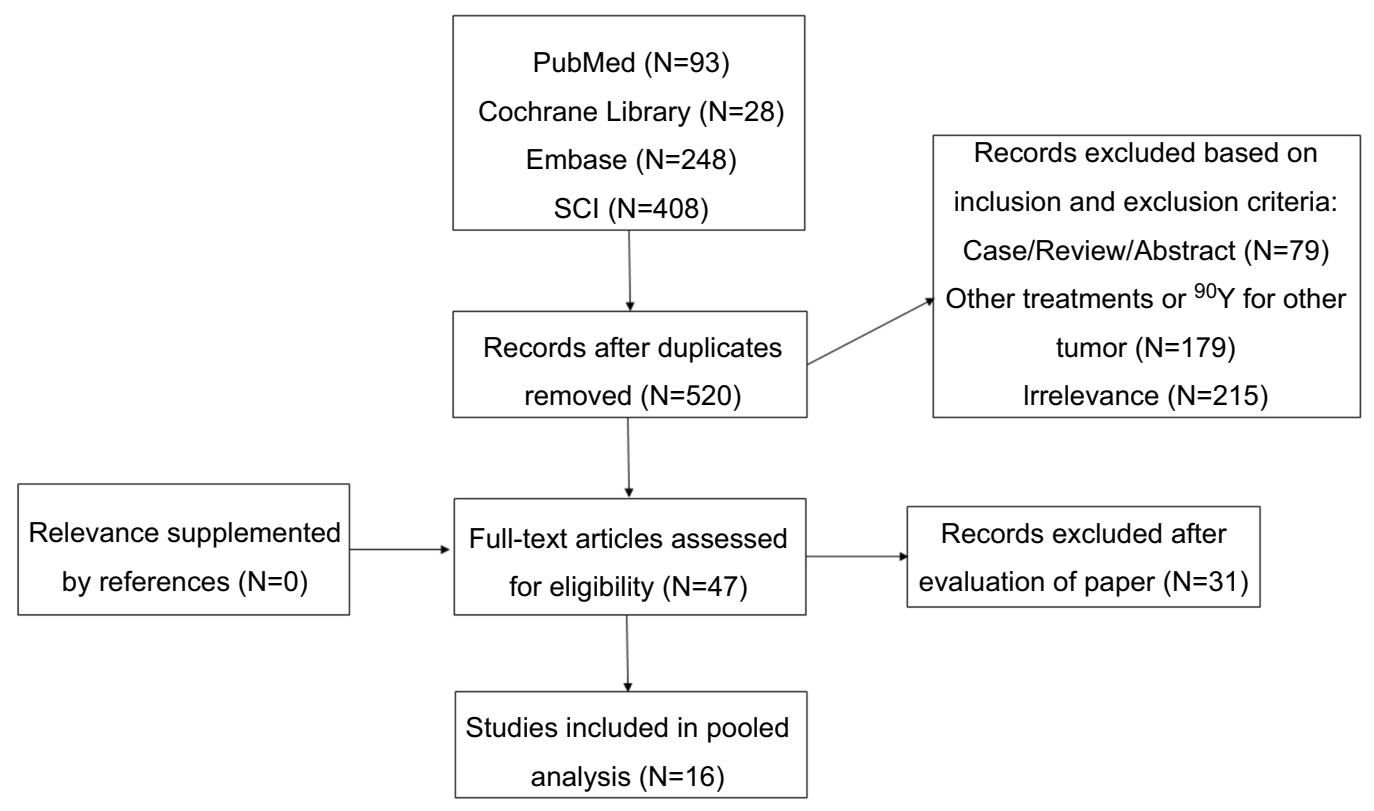

Figure I A flowchart of study identification and selection.

DCR was 77.3\% (95\%CI: $63.5-91.1 \%)$ and $77.4 \%(95 \% \mathrm{CI}$ : $66.8-87.9 \%$ ) in the glass and resin microspheres group respectively. There were six studies reporting 1-year survival rate with a median of $51.5 \%$ (range: $32.6-67.9 \%$ ).

Side effects and the proportion of grade III-IV toxicities were listed in Table 3. Clinical toxicities mainly included fatigue (median: $31.7 \%$; range: $0.0-87.5 \%$ ), anorexia (median: $10.0 \%$; range: $0.0-79.2 \%$ ), abdominal pain (median: 30.0\%; range: $0.0-85.0 \%$ ), nausea (median: $16.0 \%$; range: $0.0-62.5 \%$ ), vomiting (median: 9.0\%; range: $0.0-27.0 \%$ ), ascites (median: 10.5\%; range: 0.0-21.7\%). Biochemical toxicities were decreased albumin (median: 2.0\%; range: 0.0-9.0\%), elevated bilirubin (median: 5.7\%; range: $0.0-70.0 \%$ ), elevated alkaline phosphatase (median: 1.7\%; range: $0.0-46.0 \%$ ). The incidence of gastroduodenal ulceration was a median of $4.0 \%$ (range: $0.0-5.0 \%)$ in 5 studies reporting side effects. A median of 7.8\% (range: $0.0-25.0 \%$ ) grade III-IV toxicities (including gastroduodenal ulceration) was reported in 10 studies.

Mild and moderate heterogeneity was shown in pooled median OS and DCR. These estimates were robust in the sensitivity analysis. No significant publication bias was identified in pooled analysis.

\section{Discussion}

The pooled analysis showed that TARE with ${ }^{90} \mathrm{Y}$ microspheres can be an effective treatment for unresectable ICC with a pooled median OS of $14.3(95 \% \mathrm{CI}$ :
12.0-17.1) months. According to RECIST, the pooled DCR was $77.2 \%$ (95\%CI: 70.2-84.2\%). Subgroup analysis was conducted by microsphere type, it seems that there were similar median OS and DCR in the glass and resin microspheres group. In addition, it was associated with mild clinical and biochemical toxicities, and often these symptoms were relieved over time.

With the increasing incidence of ICC and impossibility of surgical resection, more and more people are exploring new treatments. TARE with ${ }^{90} \mathrm{Y}$ microspheres has gradually become an effective treatment by using an intraarterial injection of microspheres loaded with ${ }^{90} \mathrm{Y}$ microspheres as the source of internal radiation. ${ }^{27} \mathrm{Al}-$ A dra et al $^{9}$ reported the OS of 15.5 months in the pooled analysis for the treatment of ICC with ${ }^{90} \mathrm{Y}$ radioembolization. However, seven abstracts were included in the pooled analysis, which provided limited information regarding treatment and follow-up outcomes. In the current pooled analysis, we excluded abstracts and added literature published in recent years, which provided more comprehensive information. We came to a similar median OS of 14.3 months. Subgroup analysis was performed based on the type of microspheres, and the median OS was similar in the resin and glass microspheres groups (14.0 vs 14.3 months). Unfortunately, due to the heterogeneity of studies in each group, the random effects model was performed, which failed to compare the differences between groups. Nezami et $\mathrm{al}^{28}$ compared the dose of 


\begin{tabular}{|c|c|c|c|c|c|c|c|c|c|c|c|c|c|c|c|c|c|}
\hline \multicolumn{2}{|c|}{ 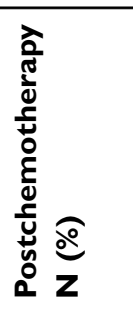 } & $\begin{array}{l}\text { a } \\
\stackrel{\infty}{d} \\
\end{array}$ & 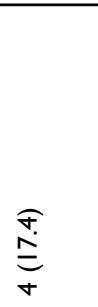 & 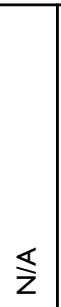 & 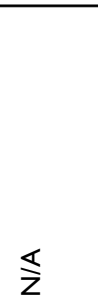 & 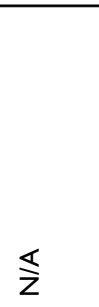 & 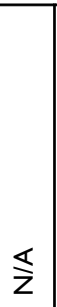 & $\overleftarrow{z}$ & 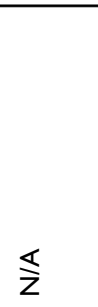 & $\stackrel{\nwarrow}{z}$ & 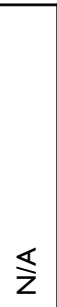 & 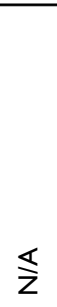 & $\widehat{\overline{\check{c}}}$ & $\stackrel{\nwarrow}{z}$ & $\overleftrightarrow{z}$ & $\underset{m}{\stackrel{0}{\ominus}}$ & 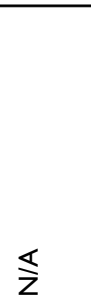 \\
\hline \multicolumn{2}{|c|}{ 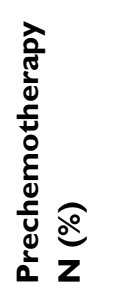 } & $\begin{array}{l}\underset{\mathrm{O}}{\mathrm{i}} \\
\underline{\infty}\end{array}$ & 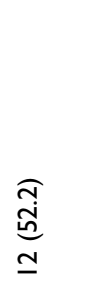 & $\begin{array}{l}o \\
\stackrel{0}{0} \\
\stackrel{0}{=} \\
\underline{a}\end{array}$ & 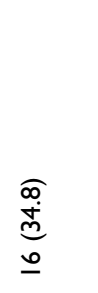 & 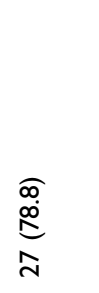 & 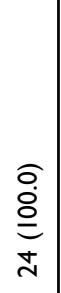 & $\begin{array}{l}\widehat{3} \\
\stackrel{0}{\circ} \\
\sigma \\
\sigma\end{array}$ & $\begin{array}{l}\frac{\pi}{a} \\
\underline{n} \\
\underline{n}\end{array}$ & 官 & $\overleftarrow{z}$ & 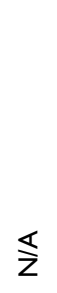 & 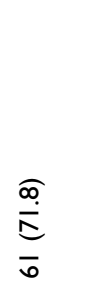 & $\begin{array}{l}\underset{\mathfrak{s}}{\infty} \\
\infty \\
\underline{\underline{n}} \\
\underline{\underline{n}}\end{array}$ & $\begin{array}{c}\overline{\bar{a}} \\
\bar{\sigma} \\
\bar{\sigma}\end{array}$ & $\underset{\substack{\mathfrak{d} \\
\text { in }}}{.}$ & 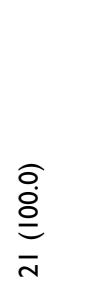 \\
\hline \multicolumn{2}{|c|}{ 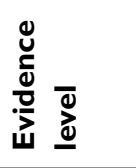 } & $\begin{array}{l}\stackrel{\square}{\pi} \\
\frac{\pi}{0} \\
\frac{0}{0} \\
\Sigma\end{array}$ & 3 & 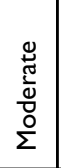 & 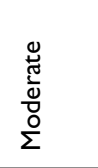 & 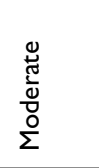 & 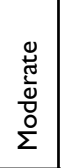 & 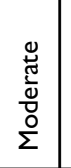 & 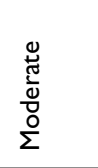 & 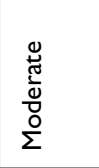 & 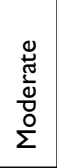 & 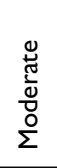 & 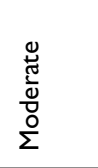 & 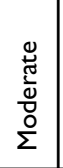 & 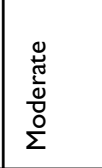 & 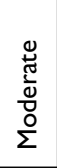 & 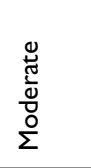 \\
\hline \multicolumn{2}{|c|}{ 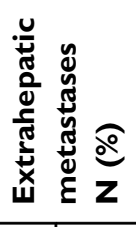 } & 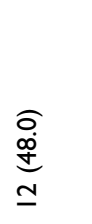 & 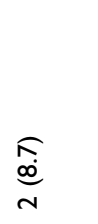 & $\begin{array}{l}\sigma \\
\stackrel{\sigma}{n} \\
\hat{\omega} \\
=\end{array}$ & 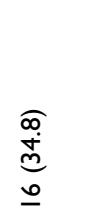 & 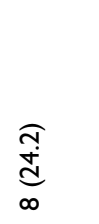 & 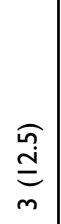 & $\frac{\widehat{m}}{\frac{m}{m}}$ & $\begin{array}{l}\text { å } \\
\stackrel{0}{0} \\
=\end{array}$ & 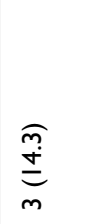 & 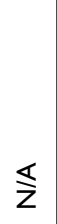 & $\stackrel{\nwarrow}{Z}$ & 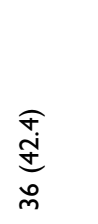 & $\begin{array}{l}\frac{\pi}{2} \\
\stackrel{d}{\sigma} \\
\sigma\end{array}$ & 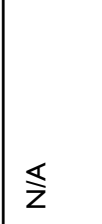 & 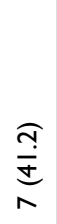 & 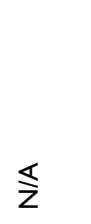 \\
\hline \multirow{4}{*}{ 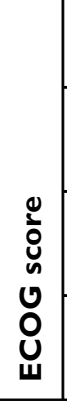 } & $m$ & 0 & 0 & 0 & 0 & 0 & 0 & 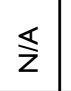 & 0 & $N$ & $\lesssim$ & $\lesssim$ & 0 & $\lesssim$ & 0 & 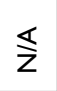 & 0 \\
\hline & $N$ & $m$ & 0 & + & - & $a$ & 0 & $\overleftarrow{z}$ & in & $\underline{\varrho}$ & $\overleftarrow{z}$ & 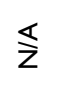 & $\stackrel{\infty}{\sim}$ & 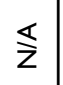 & & $\overleftarrow{z}$ & $m$ \\
\hline & - & $\lambda$ & in & \pm & $\bar{\sim}$ & $\wedge$ & $\infty$ & 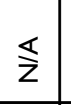 & $\underline{m}$ & $m$ & $\lesssim$ & $\lesssim$ & $\approx$ & 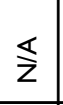 & ㅇ & 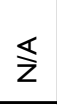 & $\infty$ \\
\hline & 0 & $\underline{\underline{n}}$ & $\underline{\infty}$ & - & $\stackrel{ \pm}{\sim}$ & $\simeq$ & $\underline{0}$ & 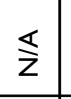 & $=$ & 0 & $\lesssim$ & 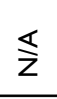 & $\stackrel{\varphi}{m}$ & $\widehat{s}$ & $\stackrel{\sim}{\sim}$ & $\overleftarrow{z}$ & $\sigma$ \\
\hline \multicolumn{2}{|c|}{$\frac{Ð}{\frac{0}{2}} \mathbf{z}$} & $\underline{\underline{0}}$ & 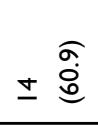 & $\begin{array}{l}\widehat{\infty} \\
\stackrel{0}{0} \\
\stackrel{0}{n} \\
\end{array}$ & 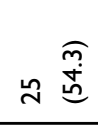 & $\underline{\substack{\mathfrak{n} \\
\dot{n}}}$ & $\begin{array}{l}\text { N } \\
\stackrel{m}{m} \\
\infty \\
\infty\end{array}$ & 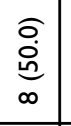 & 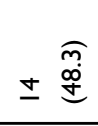 & 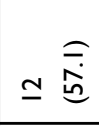 & $\overleftarrow{z}$ & $\overleftarrow{z}$ & 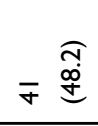 & 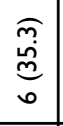 & 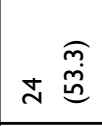 & $\begin{array}{l}\frac{\pi}{ \pm} \\
\frac{ \pm}{n} \\
\end{array}$ & $\underline{\underline{\sigma}}$ \\
\hline \multicolumn{2}{|c|}{ 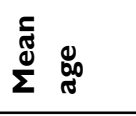 } & in & นิ & $\overbrace{\hat{\sigma}}^{m}$ & 怘 & กુ & $\frac{\infty}{\sigma}$ & 䓍 & ః & $\stackrel{n}{o}$ & 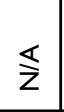 & $\stackrel{\nwarrow}{z}$ & $\stackrel{+}{\sim}$ & ڤ̆ & $\stackrel{\sigma}{\mathrm{g}}$ & $\stackrel{m}{a}$ & ત્ર \\
\hline \multicolumn{2}{|l|}{ 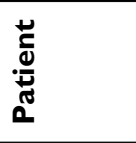 } & $\stackrel{\sim}{\sim}$ & $\ddot{\sim}$ & $\underline{\alpha}$ & fo & $\tilde{m}$ & $\stackrel{ \pm}{\sim}$ & 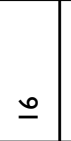 & సి & $\bar{N}$ & $\stackrel{0}{ }$ & $\stackrel{m}{m}$ & $\stackrel{\llcorner}{\infty}$ & $\simeq$ & ஜ & $\simeq$ & $\bar{N}$ \\
\hline \multicolumn{2}{|c|}{ 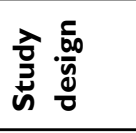 } & u & $\underset{\propto}{u}$ & U & u & $\underset{\propto}{\cup}$ & $\underset{\propto}{\cup}$ & $\underset{\propto}{U}$ & $\underset{\propto}{U}$ & $\underset{\mathscr{Y}}{U}$ & $\underset{\mathscr{Y}}{U}$ & $\underset{\propto}{U}$ & $\underset{\propto}{U}$ & U & $\underset{\mathscr{X}}{\cup}$ & $\underset{\propto}{U}$ & U \\
\hline \multicolumn{2}{|c|}{ ঠ } & 윰 & $\stackrel{\circ}{\circ}$ & $\frac{m}{\bar{i}}$ & $\frac{m}{i}$ & $\stackrel{\sim}{\bar{N}}$ & $\overline{\bar{N}}$ & $\stackrel{\circ}{\circ}$ & $\overline{\bar{\alpha}}$ & $\frac{\infty}{i}$ & $\overline{\bar{N}}$ & $\overline{\bar{i}}$ & $\frac{\infty}{2}$ & $\frac{n}{\bar{\alpha}}$ & $\stackrel{\circ}{\circ}$ & $\frac{\infty}{2}$ & $\frac{\pi}{\grave{N}}$ \\
\hline \multicolumn{2}{|l|}{ 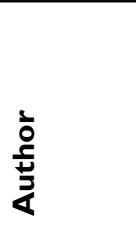 } & 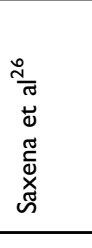 & 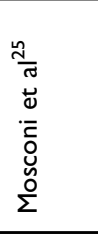 & 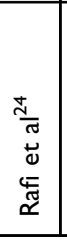 & 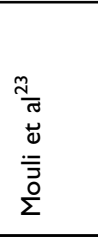 & 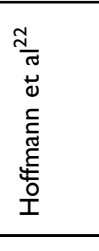 & 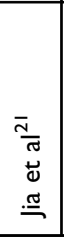 & 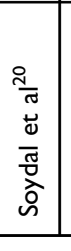 & 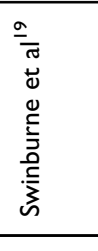 & 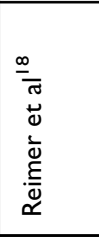 & 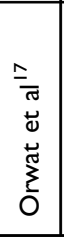 & 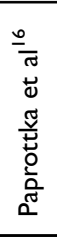 & 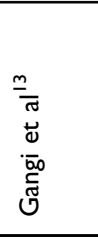 & 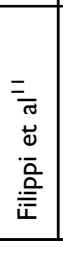 & 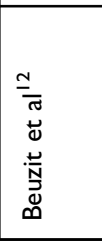 & 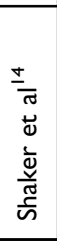 & 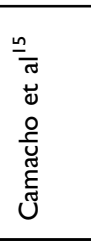 \\
\hline
\end{tabular}




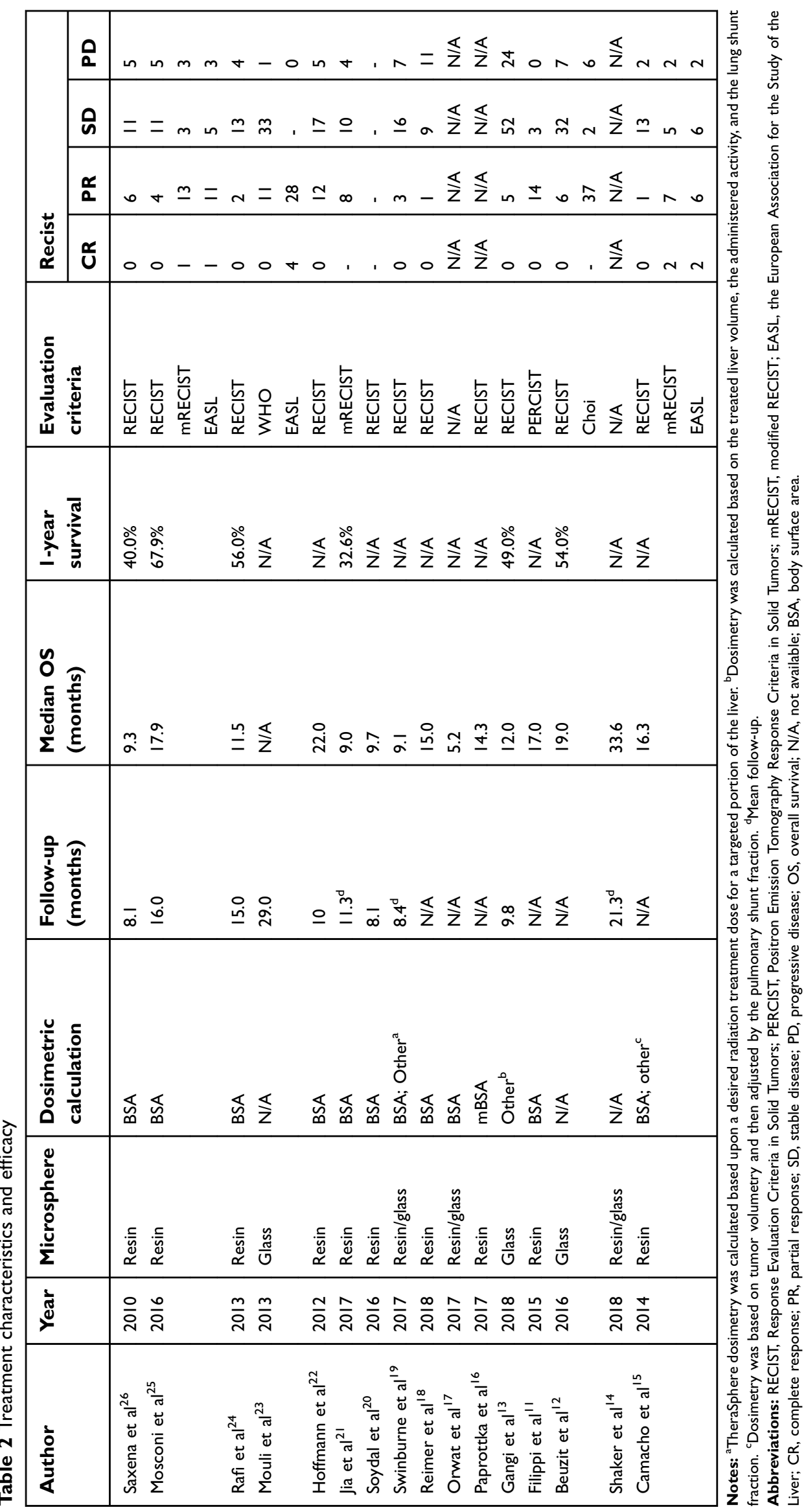


Pooled analysis of median overall survival

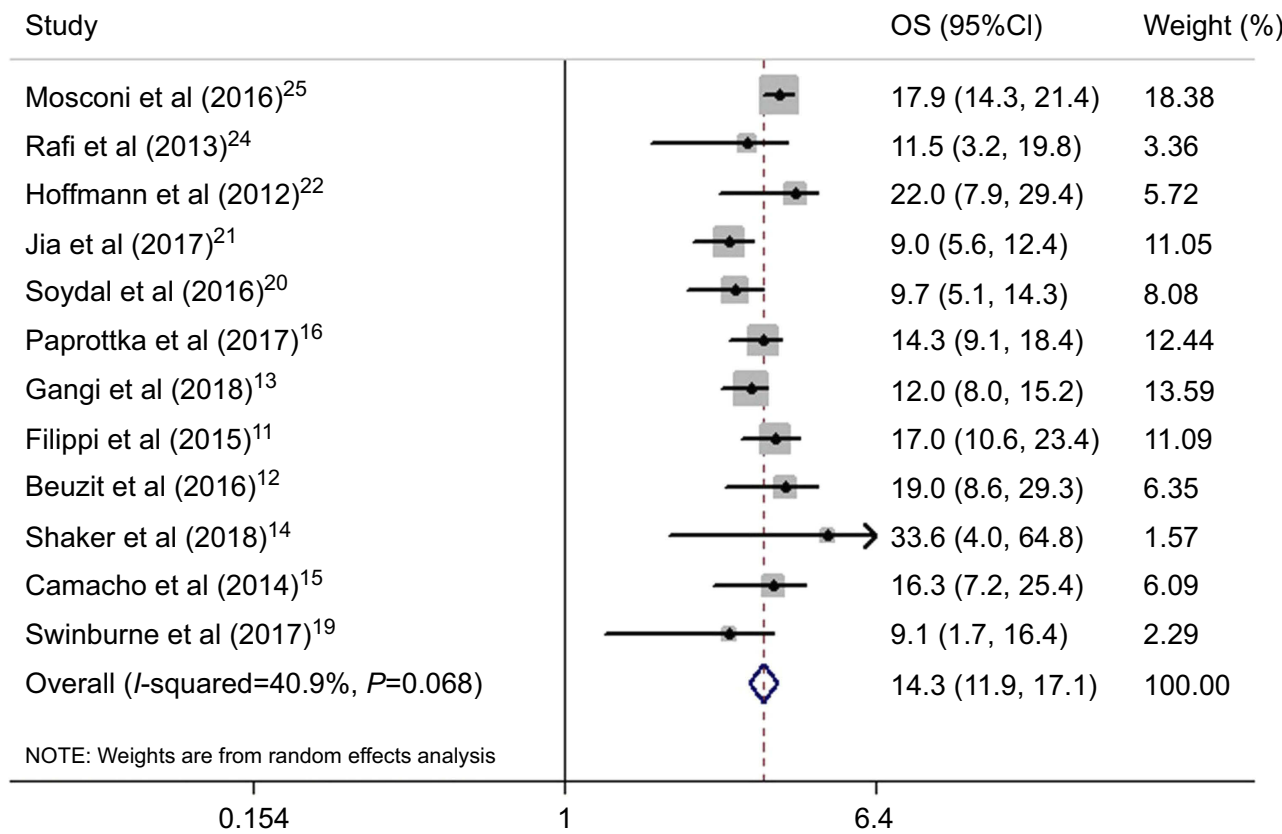

Figure 2 Pooled analysis of median overall survival. Abbreviation: OS, overall survival.

\begin{tabular}{|c|c|c|}
\hline Study & DCR $(95 \% \mathrm{Cl})$ & Weight $(\%)$ \\
\hline Saxena et al $(2010)^{26}$ & $0.773(0.598,0.948)$ & 9.46 \\
\hline Mosconi et al $(2016)^{25}$ & $0.750(0.560,0.940)$ & 8.58 \\
\hline Rafi et al $(2013)^{24}$ & $0.789(0.606,0.973)$ & 8.96 \\
\hline Hoffmann et al $(2012)^{22}$ & $0.879(0.767,0.990)$ & 14.61 \\
\hline Swinburne et al $(2017)^{19}$ & $0.731(0.560,0.901)$ & 9.76 \\
\hline Reimer et al $(2018)^{18}$ & $0.476(0.263,0.690)$ & 7.34 \\
\hline Gangi et al $(2018)^{13}$ & $0.704(0.604,0.803)$ & 15.79 \\
\hline Beuzit et al $(2016)^{12}$ & $0.844(0.739,0.950)$ & 15.15 \\
\hline Camacho et al $(2014)^{15}$ & $0.875(0.713,1.037)$ & 10.64 \\
\hline Overall $(I$-squared $=50.7 \%, P=0.039)$ & $0.772(0.702,0.842)$ & 100.00 \\
\hline NOTE: Weights are from random effects analysis & & \\
\hline 0.5 & & \\
\hline
\end{tabular}

Figure 3 Pooled analysis of disease control rate. Abbreviation: DCR, disease control rate.

radiation delivered through glass and resin-based ${ }^{90} \mathrm{Y}$ microspheres to ICC and concluded that ${ }^{90} \mathrm{Y}$ both glass and resin-based microspheres radioembolization were feasible and safe in the treatment of ICC, while glass microsphere delivers a higher dose of ${ }^{90} \mathrm{Y}$ to the targeted tumors. However, it remains to be further studied whether the two types of microspheres affect the prognosis of ICC patients. Ray et $\mathrm{al}^{29}$ reported that the pooled median OS of transarterial chemoembolization (TACE) for unresectable ICC was 13.4 months. Boehm et $\mathrm{al}^{30}$ 


\begin{tabular}{|c|c|}
\hline 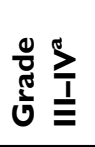 & 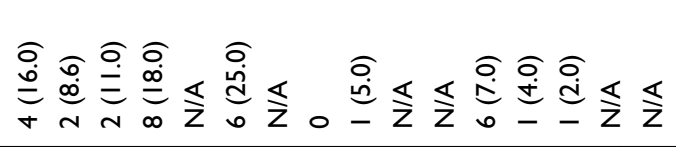 \\
\hline 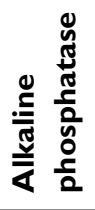 & 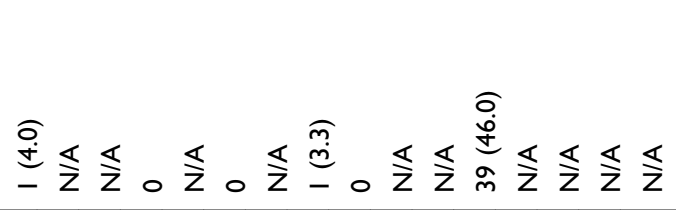 \\
\hline 产 & 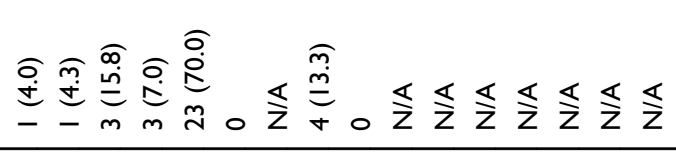 \\
\hline 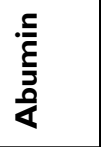 & 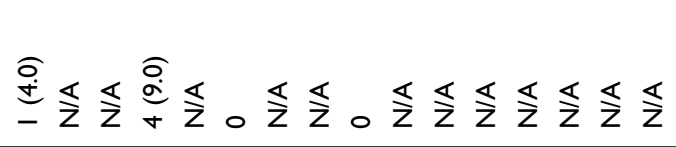 \\
\hline 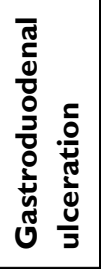 & 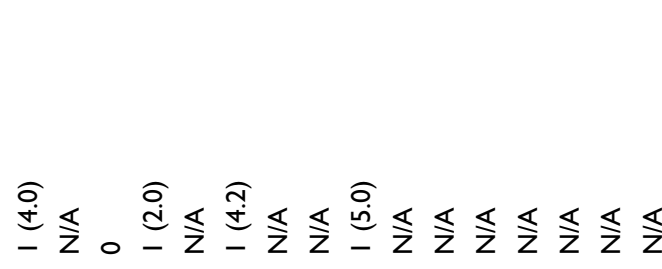 \\
\hline 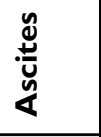 & 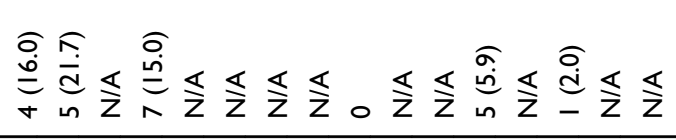 \\
\hline 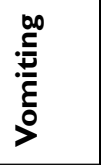 & 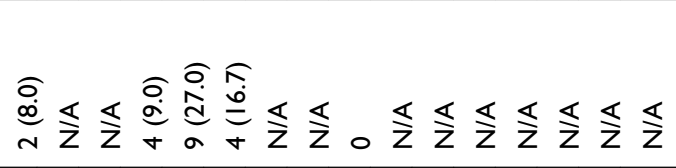 \\
\hline 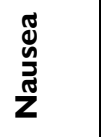 & 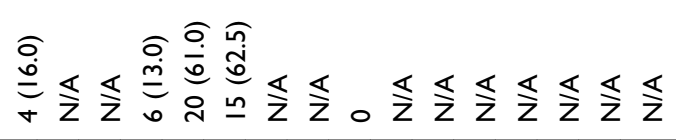 \\
\hline 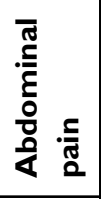 & 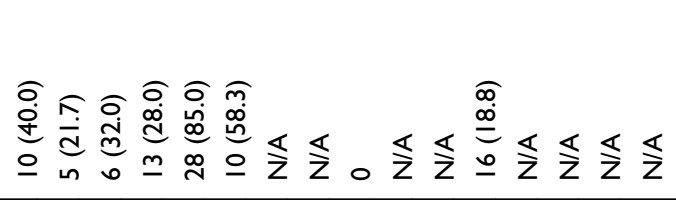 \\
\hline $\begin{array}{l}\frac{\pi}{x} \\
0 \\
\frac{0}{2} \\
\stackrel{\frac{c}{4}}{4}\end{array}$ & 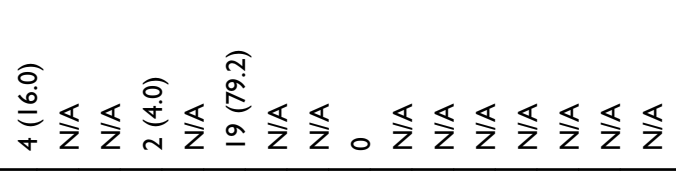 \\
\hline 离 & 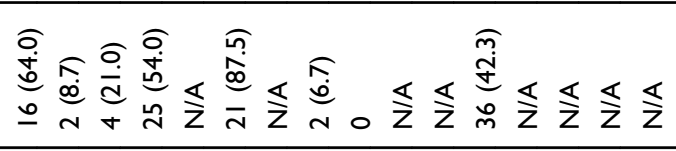 \\
\hline ঠ & 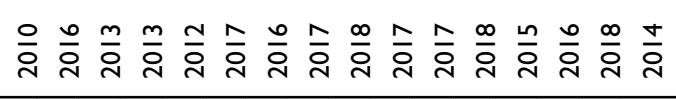 \\
\hline & 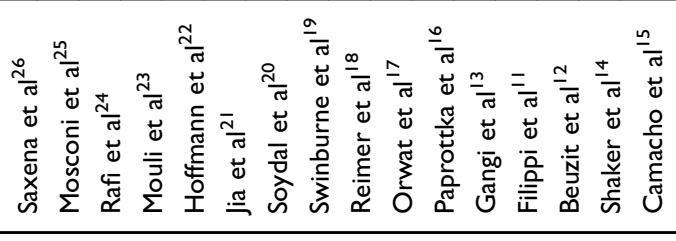 \\
\hline
\end{tabular}


conducted a pooled median OS of 12.4 months for the treatment of TACE. It seems that median OS of TARE with ${ }^{90} \mathrm{Y}$ microspheres was generally consistent with TACE. However, further randomized controlled trials are needed to confirm these results.

In the current pooled analysis, most of the studies (11/16) evaluated tumor response according to RECIST, and the pooled DCR was $77.2 \%$, which indicated that TARE was an effective treatment for ICC. However TARE with ${ }^{90} \mathrm{Y}$ microspheres usually leads to necrosis without an actual decrease of tumor size, RECIST ${ }^{31,32}$ only considers the change in the size of target lesions, and the association between RECIST and survival still needs further to be investigated. PET can evaluate the change of tumor volume through the difference of standardized uptake value, which is valuable in assessing the activity of cancer therapies that stabilize diseases. ${ }^{33}$ Zerizer et $\mathrm{al}^{34}$ reported that $18 \mathrm{~F}-\mathrm{FDG}$ PET-CT was superior to RECIST in evaluating early response of TARE and predicting progression free survival in patients with liver metastases. Therefore, PET-based approaches are expected to be effective evaluation criteria in tumor response after TARE with ${ }^{90} \mathrm{Y}$ microspheres.

In addition, TARE with ${ }^{90} \mathrm{Y}$ microspheres is associated with some side effects. In the current pooled analysis, the common clinical toxicities mainly included fatigue, abdominal pain, nausea, vomiting, ascites, and biochemical toxicities had decreased albumin, elevated bilirubin and alkaline phosphatase, etc. These side effects were usually mild and acceptable, and could be resolved without medical therapy. Moreover, gastroduodenal ulceration is a relatively common serious side effect of TARE with ${ }^{90} \mathrm{Y}$ microspheres, ${ }^{35}$ which is caused by nontargeted microsphere distribution, so it is necessary to clarify the vascular anatomy and undergo prophylactic arterial embolization; in addition, microspheres must be carefully injected during the treatment process to avoid nontargeted embolization.

There are several limitations in the current pooled analysis. First, in the pooled analysis, not all studies reported the population and treatment characteristics that were meta-analyzed, thus not allowing a complete analysis of heterogeneity sources. Second, meta-regression was not performed in the current analysis because the pooled results were robust in the sensitivity analysis, which suggested the source of heterogeneity may not exist in studies, but in individuals. Third, side effects were summarized only as descriptive words, standardized methodology needs to be used. Fourth, the current results failed to help define the best population for TARE, but this pooled analysis included the best available evidence and provided valuable information on the therapeutic efficacy and safety of TARE with ${ }^{90} \mathrm{Y}$ microspheres for unresectable ICC.

\section{Conclusion}

TARE with ${ }^{90} \mathrm{Y}$ microspheres is a promising therapeutic option for patients with unresectable ICC with acceptable side effects. The different microspheres seem to have no influence on therapeutic efficacy, and TARE with ${ }^{90} \mathrm{Y}$ microspheres has a similar OS compared with TACE reported in previous studies. A large sample of randomized controlled trial is warranted to confirm the above results.

\section{Acknowledgments}

We would like to thank all our colleagues and authors who cooperated with us by preparing the full text of the papers. The authors received no financial support for the research, authorship, and/or publication of this article.

\section{Author contributions}

All authors contributed to data analysis, drafting and revising the article, gave final approval of the version to be published, and agree to be accountable for all aspects of the work.

\section{Disclosure}

The authors report no conflicts of interest in this work.

\section{References}

1. Endo I, Gonen M, Yopp AC, et al. Intrahepatic cholangiocarcinoma. Ann Surg. 2008;248(1):84-96. doi:10.1097/SLA.0b013e318176c4d3

2. Hogdall D, O'Rourke CJ, Taranta A, Oliveira DV, Andersen JB. Molecular pathogenesis and current therapy in intrahepatic cholangiocarcinoma. Dig Dis. 2016;34(4):440-451. doi:10.1159/ 000444562

3. Ellis MC, Cassera MA, Vetto JT, Orloff SL, Hansen PD, Billingsley KG. Surgical treatment of intrahepatic cholangiocarcinoma: outcomes and predictive factors. Hpb. 2011;13(1):59-63. doi:10.1111/j.14772574.2010.00242.x

4. Hong K, Geschwind J-FH. Locoregional intra-arterial therapies for unresectable intrahepatic cholangiocarcinoma. Semin Oncol. 2010;37 (2):110-117. doi:10.1053/j.seminoncol.2010.03.002

5. Yang L, Shan J, Shan L, Saxena A, Bester L, Morris DL. Trans-arterial embolisation therapies for unresectable intrahepatic cholangiocarcinoma: a systematic review. J Gastrointest Oncol. 2015;6(5):570-588. doi:10.3978/j.issn.2078-6891.2015.055

6. Valle JW, Furuse J, Jitlal M, et al. Cisplatin and gemcitabine for advanced biliary tract cancer: a meta-analysis of two randomised trials. Ann Oncol. 2013;25(2):391-398. doi:10.1093/annonc/mdt540

7. Koay EJ, Odisio BC, Javle M, Vauthey J-N, Crane CH. Management of unresectable intrahepatic cholangiocarcinoma: how do we decide among the various liver-directed treatments? Hepatobiliary Surg Nutr. 2017;6(2):105-116. doi:10.21037/hbsn.2017.01.16 
8. Zheng J, Irani Z, Lawrence D, Flaherty K, Arellano RS. Combined effects of Yttrium-90 transarterial radioembolization around immunotherapy for hepatic metastases from uveal melanoma: a preliminary retrospective case series. J Vasc Interv Radiol. 2018;29 (10):1369-1375. doi:10.1016/j.jvir.2018.04.030

9. Al-Adra DP, Gill RS, Axford SJ, Shi X, Kneteman N, Liau -S-S. Treatment of unresectable intrahepatic cholangiocarcinoma with yttrium-90 radioembolization: a systematic review and pooled analysis. Eur J Surg Oncol (EJSO). 2015;41(1):120-127. doi:10.1016/j.ejso.2014.09.007

10. Sara H, Downs NB. The feasibility of creating a checklist for the assessment of the methodological quality both of randomised and non-randomised studies of health care interventions. J Epidemiol Community Health. 1998;52(6):377-384.

11. Filippi L, Pelle G, Cianni R, Scopinaro F, Bagni O. Change in total lesion glycolysis and clinical outcome after (90)Y radioembolization in intrahepatic cholangiocarcinoma. Nucl Med Biol. 2015;42 (1):59-64. doi:10.1016/j.nucmedbio.2014.08.011

12. Beuzit L, Edeline J, Brun V, et al. Comparison of Choi criteria and response evaluation criteria in solid tumors (RECIST) for intrahepatic cholangiocarcinoma treated with glass-microspheres Yttrium-90 selective internal radiation therapy (SIRT). Eur J Radiol. 2016;85 (8):1445-1452. doi:10.1016/j.ejrad.2016.05.020

13. Gangi A, Shah J, Hatfield N, et al. Intrahepatic cholangiocarcinoma treated with transarterial Yttrium-90 glass microsphere radioembolization: results of a single institution retrospective study. J Vasc Interventional Radiol. 2018;29(8):1101-1108. doi:10.1016/j.jvir.2018.04.001

14. Shaker TM, Chung C, Varma MK, et al. Is there a role for Ytrrium-90 in the treatment of unresectable and metastatic intrahepatic cholangiocarcinoma? Am J Surg. 2018;215(3):467-470. doi:10.1016/j.amjsurg.2017.11.022

15. Camacho JC, Kokabi N, Xing M, Prajapati HJ, El-Rayes B, Kim HS. Modified response evaluation criteria in solid tumors and European Association for the study of the liver criteria using delayed-phase imaging at an early time point predict survival in patients with unresectable intrahepatic cholangiocarcinoma following Yttrium-90 radioembolization. $J$ Vasc Interventional Radiol. 2014;25 (2):256-265. doi:10.1016/j.jvir.2013.10.056

16. Paprottka KJ, Schoeppe F, Ingrisch M, et al. Pre-therapeutic factors for predicting survival after radioembolization: a single-center experience in 389 patients. Eur J Nucl Med Mol Imaging. 2017;44 (7):1185-1193. doi:10.1007/s00259-017-3646-z

17. Orwat KP, Beckham TH, Cooper SL, et al. Pretreatment albumin may aid in patient selection for intrahepatic Y-90 microsphere transarterial radioembolization (TARE) for malignancies of the liver. J Gastrointest Oncol. 2017;8(6):1072-1078. doi:10.21037/jgo.2017.06.18

18. Reimer P, Virarkar MK, Binnenhei M, Justinger M, Schön MR, Tatsch K. Prognostic factors in overall survival of patients with unresectable intrahepatic cholangiocarcinoma treated by means of Yttrium-90 radioembolization: results in therapy-naive patients. Cardiovasc Intervent Radiol. 2018;41(5):744-752. doi:10.1007/s00270-017-1871-2

19. Swinburne NC, Biederman DM, Besa C, et al. Radioembolization for unresectable intrahepatic cholangiocarcinoma: review of safety, response evaluation criteria in solid tumors 1.1 imaging response and survival. Cancer Biother Radiopharm. 2017;32(5):161-168. doi:10.1089/cbr.2017.2189

20. Soydal C, Kucuk ON, Bilgic S, Ibis E. Radioembolization with (90)Y resin microspheres for intrahepatic cholangiocellular carcinoma: prognostic factors. Ann Nucl Med. 2016;30(1):29-34. doi:10.1007/ s12149-015-1026-y

21. Jia Z, Paz-Fumagalli R, Frey G, Sella DM, McKinney JM, Wang W. Resin-based Yttrium-90 microspheres for unresectable and failed first-line chemotherapy intrahepatic cholangiocarcinoma: preliminary results. J Cancer Res Clin Oncol. 2017;143(3):481-489. doi:10.1007/ s00432-016-2291-4
22. Hoffmann RT, Paprottka PM, Schon A, et al. Transarterial hepatic yttrium-90 radioembolization in patients with unresectable intrahepatic cholangiocarcinoma: factors associated with prolonged survival. Cardiovasc Intervent Radiol. 2012;35(1):105-116. doi:10.1007/ s00270-011-0142-x

23. Mouli S, Memon K, Baker T, et al. Yttrium-90 radioembolization for intrahepatic cholangiocarcinoma: safety, response, and survival analysis. J Vasc Interv Radiol. 2013;24(8):1227-1234. doi:10.1016/ j.jvir.2013.02.031

24. Rafi S, Piduru SM, El-Rayes B, et al. Yttrium-90 radioembolization for unresectable standard-chemorefractory intrahepatic cholangiocarcinoma: survival, efficacy, and safety study. Cardiovasc Intervent Radiol. 2013;36(2):440-448. doi:10.1007/s00270-0120463-4

25. Mosconi C, Gramenzi A, Ascanio S, et al. Yttrium-90 radioembolization for unresectable/recurrent intrahepatic cholangiocarcinoma: a survival, efficacy and safety study. $B r \quad J$ Cancer. 2016;115 (3):297-302. doi:10.1038/bjc.2016.191

26. Saxena A, Bester L, Chua TC, Chu FC, Morris DL. Yttrium-90 radiotherapy for unresectable intrahepatic cholangiocarcinoma: a preliminary assessment of this novel treatment option. Ann Surg Oncol. 2010;17(2):484-491. doi:10.1245/s10434-009-0777-x

27. Cristina Mosconi AC, Ascanio S. Yttrium-90 microsphere radioembolization in unresectable intrahepatic cholangiocarcinoma. Future Oncol. 2017;13(15):1301-1310. doi:10.2217/fon-20170022

28. Nezami N, Kokabi N, Camacho JC, Schuster DM, Xing M, Kim HS. (90)Y radioembolization dosimetry using a simple semi-quantitative method in intrahepatic cholangiocarcinoma: glass versus resin microspheres. Nucl Med Biol. 2018;59:22-28. doi:10.1016/j. nucmedbio.2018.01.001

29. Ray CE Jr., Edwards A, Smith MT, et al. Metaanalysis of survival, complications, and imaging response following chemotherapy-based transarterial therapy in patients with unresectable intrahepatic cholangiocarcinoma. J Vasc Interv Radiol. 2013;24(8):1218-1226. doi:10.1016/j.jvir.2013.03.019

30. Boehm LM, Jayakrishnan TT, Miura JT, et al. Comparative effectiveness of hepatic artery based therapies for unresectable intrahepatic cholangiocarcinoma. J Surg Oncol. 2015;111(2):213-220. doi:10.1002/jso.23781

31. Wahl RL, Jacene H, Kasamon Y, Lodge MA. From RECIST to PERCIST: evolving considerations for PET response criteria in solid tumors. J Nucl Med. 2009;50(Suppl 1):122S-150S. doi:10.2967/jnumed.108.057307

32. Kim MN, Kim BK, Han KH, Kim SU. Volution from WHO to EASL and mRECIST for hepatocellular carcinoma: considerations for tumor response assessment. Expert Rev Gastroenterol Hepatol. 2015;9(3):335-348. doi:10.1586/17474124.2015.959929

33. Haug AR, Heinemann V, Bruns CJ, et al. 18F-FDG PET independently predicts survival in patients with cholangiocellular carcinoma treated with $90 \mathrm{Y}$ microspheres. Eur J Nucl Med Mol Imaging. 2011;38(6):1037-1045. doi:10.1007/s00259-011-1736-x

34. Zerizer I, Al-Nahhas A, Towey D, et al. The role of early ${ }^{18} \mathrm{~F}-\mathrm{FDG}$ $\mathrm{PET} / \mathrm{CT}$ in prediction of progression-free survival after ${ }^{90} \mathrm{Y}$ radioembolization: comparison with RECIST and tumour density criteria. Eur J Nucl Med Mol Imaging. 2012;39(9):1391-1399. doi:10.1007/ s00259-012-2149-1

35. Lam MGEH, Banerjee S, Louie JD, et al. Root cause analysis of gastroduodenal ulceration after Yttrium-90 Radioembolization. Cardiovasc Intervent Radiol. 2013;36(6):1536-1547. doi:10.1007/ s00270-013-0579-1 


\section{Publish your work in this journal}

OncoTargets and Therapy is an international, peer-reviewed, open access journal focusing on the pathological basis of all cancers, potential targets for therapy and treatment protocols employed to improve the management of cancer patients. The journal also focuses on the impact of management programs and new therapeutic agents and protocols on patient perspectives such as quality of life, adherence and satisfaction. The manuscript management system is completely online and includes a very quick and fair peer-review system, which is all easy to use. Visit http://www.dovepress.com/ testimonials.php to read real quotes from published authors.

Submit your manuscript here: https://www.dovepress.com/oncotargets-and-therapy-journal 